\title{
Rabaska
}

Revue d'ethnologie de l'Amérique française

PRICE, JEREMY, LICIA BAgINI et MARLÈnE BELLY [dir.]. Langue, musique, identité. Actes du colloque tenu à Poitiers du 21 au 23

novembre 2007. Paris, Publibook, 2011, 252 p. ISBN

978-2-7483-7063-8

\section{Marie-Hélène Pichette}

Volume 12, 2014

URI : https://id.erudit.org/iderudit/1026820ar

DOI : https://doi.org/10.7202/1026820ar

Aller au sommaire du numéro

Éditeur(s)

Société québécoise d'ethnologie

ISSN

1703-7433 (imprimé)

1916-7350 (numérique)

Découvrir la revue

Citer ce compte rendu

Pichette, M.-H. (2014). Compte rendu de [PRICE, JEREMY, LICIA BAgINI et MARLÈNE Belly [dir.]. Langue, musique, identité. Actes du colloque tenu à Poitiers du 21 au 23 novembre 2007. Paris, Publibook, 2011, 252 p. ISBN 978-2-7483-7063-8]. Rabaska, 12, 296-298. https://doi.org/10.7202/1026820ar

Ce document est protégé par la loi sur le droit d'auteur. L'utilisation des services d'Érudit (y compris la reproduction) est assujettie à sa politique d'utilisation que vous pouvez consulter en ligne.

https://apropos.erudit.org/fr/usagers/politique-dutilisation/ 
liées à l'interdépendance du domaine culturel au monde politique. En effet, John R. Porter insiste à plusieurs reprises sur les différentes interventions qu'il a menées auprès du gouvernement afin de repositionner favorablement l'institution qu'il représentait. M. Porter démontre alors qu'il est nécessaire pour tout gestionnaire culturel de connaître ses forces et de comprendre son opposant, pour ainsi positionner efficacement chacune de ses actions comme un pion sur l'échiquier.

Pour conclure, Devenir un leader culturel est un ouvrage éclairant puisqu'il jette un regard nouveau sur le milieu muséal et culturel, mais tout particulièrement sur ceux qui y œuvrent. En effet, ce livre souligne l'importance de ces travailleurs culturels, qui marquent littéralement de leurs personnalités les institutions dans lesquelles ils s'investissent. En étudiant le parcours de M. Porter, nous constatons que les nécessités du domaine muséal et culturel ont changé, évolué et que ces professionnels doivent constamment progresser en accord avec eux. Enfin, par les nombreux défis relevés au cours de sa carrière, John R. Porter montre bien qu'il faut parfois faire preuve de leadership, de créativité et d'audace pour devenir un acteur de changement.

Marie-Ève Goulet

Université du Québec à Montréal

Price, Jeremy, Licia Bagini et Marlène Belly [dir.]. Langue, musique, identité. Actes du colloque tenu à Poitiers du 21 au 23 novembre 2007. Paris, Publibook, 2011, 252 p. ISBN 978-2-7483-7063-8.

L'ouvrage Langue, musique, identité est le produit du colloque du même nom tenu à l'Université de Poitiers en novembre 2007. Il regroupe, en cinq grandes thématiques, les communications de quinze ethnomusicologues, germanistes, linguistes et autres chercheurs. À une époque où le lien entre musique et identité culturelle paraît évident, plusieurs chercheurs s'intéressent maintenant au " phénomène du son en tant que facteur décisif pour les différentes pratiques culturelles et constructions identitaires (p. 15)».

La première partie intitulée "Constructions identitaires et systèmes de valeurs » s'ouvre sur le texte de Marlène Belly qui aborde le lien entre musique et spiritualité dans la construction identitaire. Elle prend en exemple les musiques d'Afrique subsaharienne, celles des monastères tibétains et celles du gamelan pour montrer les rapprochements entre ces systèmes de valeur et l'organisation des sons. Monique Desroches s'intéresse ensuite à la musique des descendants des Tamouls en Martinique et à l'île de la Réunion. Elle « cherche à montrer que la musique et la religion constituent des 
points d'ancrage exceptionnels, des marques distinctives privilégiées qui agissent comme de véritables signatures dans les processus de construction identitaire » (p. 44). De son côté, Hélène Yèche analyse l'œuvre du chansonnier allemand Wolf Biermann. Son exil forcé à l'ouest le mène vers une « insatiable quête de soi et du monde » (p. 70) reflétée dans ses chansons. L'article de Jean-Charles Khalifa clôt cette section. En étudiant un corpus de mille six cents chansons de musique country américaine des années trente à cinquante, l'auteur « tent[e] de repérer [...] les sources possibles du conservatisme » (p. 82) dans une musique qui se veut ancrée dans un mouvement de contestation.

Dans la deuxième partie, "Esthétique littéraire et musicale », Andrea Allerkamp analyse les réflexions sur la musique et le langage de la poétesse et nouvelliste autrichienne Ingeborg Bachmann et les met en relation avec les textes de Theodor W. Adorno. «Il est alors question de montrer l'effort de formuler un nouveau langage afin de faire parler un silence lourd régnant sur le passé totalitaire jusqu'aux années soixante en Allemagne et en Autriche et cela grâce au processus dialectique entre langue et musique » (p. 17). Bernard Banoun s'intéresse ensuite au compositeur Paul Dessau, un des plus fidèles aux principes de Brech. Il explique comment, chez Dessau, son discours musical élaboré possède sa cohérence interne en prenant en exemple la musique dans l'opéra Lucullus. Enfin, Martin Rass constate que notre univers sonore ambiant est sursaturé. Ceci joue non seulement sur notre réception auditive, mais aussi sur la production musicale, entre autre celle des DJ.

La troisième partie traite du « renouveau des formes traditionnelles » dans trois milieux : la France, l'Angleterre et l'Italie. D'abord, Luc CharlesDominique « rappelle que le mouvement de renouveau des musiques et danses traditionnelles en France, depuis les années 1970, a réhabilité de nombreuses pratiques instrumentales déclarées traditionnelles dont certaines sont fortement territorialisées et possèdent un statut emblématique » (p. 17). Ces nouveaux emblèmes issus du revival français ont permis la revendication d'une identité culturelle régionale autre (p. 138). En Angleterre, Jeremy Price reconnaît quatre grandes périodes de revival qui correspondent «à des périodes de remise en question identitaire provoquées soit par d'importantes restructurations institutionnelles, soit par de grands conflits » (p. 155). Il explique ceci en ordre chronologique. En Italie, Licia Bagini traite de la tarentelle, cette « forme musicale dans laquelle musique et danse sont indissociables » (p. 165) du sud de l'Italie. Elle montre comment elle s'ouvre aux expériences, aux apports culturels extérieurs pour s'enrichir tout en se demandant si une telle ouverture ne risque pas de changer le sens de la tarentelle. 
« Hybridation et identité » nous présente deux cas de communautés créoles. D'abord Claude Chastagner traite de la musique zydeco chez les Créoles de couleur du sud-ouest de la Louisiane. Il constate que cette musique est rarement jouée par des gens extérieurs à la communauté et s'interroge donc sur le rapport musique zydeco, langue française et identité créole louisianaise. De son côté, David Khatilé s'intéresse à la haute-taille, ce « modèle martiniquais de contredanse française » (p. 193). Il s'interroge sur les enjeux identitaires liés à la redécouverte de cette danse.

La dernière partie s'intitule « Altérité en langue et musique ». Isabella Maria Zoppi analyse d'un point de vue littéraire, le répertoire de Paolo Conte, auteur-compositeur de chansons à texte italien. Elle constate que « le discours de Conte, basé sur une alternance de significations profondes et de désirs, se déroule autour de deux questions principales : quoi raconter et comment le raconter » (p. 211). Elle étudie le répertoire sous cet angle. Liliane Jagueneau aborde ensuite le phénomène de l'interaction entre le français et la langue régionale dans les publications de musique traditionnelle du Centre-Ouest de la France. Elle relève les éléments pouvant être considérés régionaux, puis tente d'établir les rapports entre ces langues dans les textes pour mettre en évidence la revendication d'une altérité. Jean-Jacques Castéret termine cet ouvrage en explorant la chanson et la pratique vocale en Béarn et en mettant de l'avant ses préoccupations musicales et linguistiques auprès des chanteurs de tradition.

Somme toute, plusieurs articles amènent des points de vue intéressants sur le phénomène du son dans la construction identitaire. Qu'il s'agisse de montrer à quel point musique et religion jouent un rôle important dans ces processus ou comment des renouveaux musicaux peuvent transformer une pratique musicale, ces questions continuent à susciter l'intérêt. Langue, musique, identité apporte ainsi un autre regard sur l'interaction de ces deux disciplines dans la construction identitaire.

Marie-Hélène Pichette

Université de Montréal/International School of Ouagadougou

Rousseau, Nicole et Johanne Daigle. Infirmières de colonie. Soins et médicalisation dans les régions du Québec, 1932-1972. Québec, Les Presses de 1'Université Laval, 2013, 459 p. ISBN978-2-7637-1968-9.

Comment se fait-il que les infirmières de colonie n'aient pas été l'objet d'une étude approfondie plus tôt ? C'est la première réflexion qui m'est venue en 